\title{
Comparaison du pH et du pouvoir tampon dans le rumen des ovins et des dromadaires
}

\author{
T Khorchani 1, D Demeyer 2, M Hammadi 1 \\ IInstitut des Régions Arides, 4119 Medenine, Tunisie ; 2 Vakgroep Dierlijke Productie, \\ Proefhoevestraat 10, 9090 Melle, Belgique
}

Les dromadaires sont considérés parmi les espèces les plus adaptées aux milieux arides. Ils peuvent valoriser des zones marginales et digérer relativement mieux que les ovins et même que les caprins la végétation lignifiée. La plupart des études récentes sur le rumen des ovins et des dromadaires rapportent un pouvoir tampon plus élevé chez ces derniers.

Des échantillons de contenu du rumen de 45 moutons et du premier compartiment de 33 dromadaires ont été prélevés dans deux abattoirs du Sud Est Tunisien. Les régimes alimentaires des deux espèces étaient variés, mais d'après l'aspect du contenu ruminal, le régime à base de paille de graminées était le plus fréquent. La durée du jeûne des animaux avant l'abattage n'était pas contrôlable.

Le contenu de rumen prélevé a été subdivisé en deux parties : la première a servi à la détermination immédiate $\mathrm{du} \mathrm{pH}$, ensuite elle a été filtrée puis acidifiée pour la détermination quantitative et qualitative des acides gras volatils (AGV) par chromatographie en phase gazeuse. La seconde partie a été directement versée dans des bouteilles contenant $10 \mathrm{ml}$ d'huile de paraffine pour éviter la perte du $\mathrm{CO}_{2}$, puis congelée en vue de la détermination du pouvoir tampon entre le $\mathrm{pH}=5$ et le $\mathrm{pH}=6$ $\left(\beta_{5-6}\right)$ ainsi qu'entre le $\mathrm{pH}=6$ et le $\mathrm{pH}=7\left(\beta_{6-7}\right)$ par titrimétrie selon Emmanuel et al $(1969, \mathrm{Br} J$ Nutri, 23, 805-811). Une analyse de variance à un seul facteur (espèce) a été appliquée à toutes les variables étudiées.

Le $\mathrm{pH}$ du contenu du rumen des dromadaires a été significativement plus élevé comparé à celui des moutons bien que les teneurs en AGV aient été comparables entre les deux espèces. Ceci suggère un pouvoir tampon dans le rumen plus élevé chez les dromadaires. Toutefois, la méthode titrimétrique n'a pas confirmé cette différence entre les deux espèces. Les proportions des acides acétique, propionique et butyrique ont été équivalentes chez les deux espèces étudiées.

En conclusion, bien que la méthode titrimétrique n'ait pas mis en évidence de différence du pouvoir tampon du contenu de rumen entre dromadaires et moutons, l'analyse de la relation entre le $\mathrm{pH}$ et les concentrations des AGV montre une différence entre les deux espèces, ce qui favorise une meilleure activité microbienne chez les dromadaires, nécessaire à la digestion des parois végétales selon Stewart (1977, App Env Microbiol, 33, 497-502).

\begin{tabular}{cccccccc}
$\begin{array}{c}\text { Paramètres Chimiques } \\
\text { du rumen }\end{array}$ & $\mathrm{pH}$ & $\begin{array}{c}\mathrm{AGV} \\
(\mathrm{mmol} / \mathrm{l})\end{array}$ & $\% \mathrm{C} 2$ & $\% \mathrm{C} 3$ & $\% \mathrm{C} 4$ & $\begin{array}{c}\beta_{5-6} \\
(\mathrm{mEq} / \mathrm{l})\end{array}$ & $\begin{array}{c}\beta_{6-7} \\
(\mathrm{mEq} / \mathrm{l})\end{array}$ \\
\hline $\begin{array}{c}\text { dromadaires } \\
\text { ovins }\end{array}$ & $6,63^{\mathrm{a}}$ & $72,2^{\mathrm{a}}$ & $71,6^{\mathrm{a}}$ & $19,8^{\mathrm{a}}$ & $8,6^{\mathrm{a}}$ & $29,10^{\mathrm{a}}$ & $21,07^{\mathrm{a}}$ \\
& $6,38^{\mathrm{b}}$ & $68,3^{\mathrm{a}}$ & $70,4^{\mathrm{a}}$ & $20,2^{\mathrm{a}}$ & $9,4^{\mathrm{a}}$ & $33,17^{\mathrm{a}}$ & $22,92^{\mathrm{a}}$
\end{tabular}

Dans une même colonne : $a \neq b: P<0,05$ 